\title{
Patient-Reported Outcome of a Multidisciplinary Pain Management Program, Focusing on Occupational Performance and Satisfaction with Performance
}

\author{
Kersti Samuelsson ${ }^{*}, 1$, Ulla Carlberg ${ }^{2}$, Malin Hesselstrand ${ }^{2}$, Elisabet Ölander ${ }^{2}$ and Ewa Wressle ${ }^{3}$ \\ ${ }^{I}$ Department of Clinical and Experimental Medicine, Faculty of Health Sciences, Linköping University, 58185 \\ Linköping, and Clinical Department of Rehabilitation Medicine, University Hospital, 58185 Linköping, Sweden \\ ${ }^{2}$ Rehabilitation Medicine, Faculty of Health Sciences, Department of Clinical and Experimental Medicine, Linköping \\ University, 58185 Linköping, and Pain and rehabilitation Centre, University Hospital, 58185 Linköping, Sweden \\ ${ }^{3}$ Department of Geriatrics, Faculty of Health Sciences, Linköping University, and Department of Geriatrics, University \\ Hospital, 58185 Linköping, Sweden
}

\begin{abstract}
Aim: The aim was to describe the effect of a multidisciplinary pain management program, in terms of patientreported occupational performance and satisfaction with performance.

Methods: The study is a retrospective, case series study. Data from interviews documented routinely in patient medical records were used. Interviews were made at introduction, on conclusion and six months after a pain management program. Data from all participants $(\mathrm{n}=85)$ introduced during one year, were analysed. The Canadian Occupational Performance Measure (COPM) was used as the main outcome measure.

Results: Estimated occupational performance as well as satisfaction with performance improved between measures (occupational performance $\mathrm{p}<0.001$; satisfaction with performance $\mathrm{p}<0.001$ ). The percentage of participants, who improved two or more points on the COPM ten-point scale between baseline and the 6-month follow up, was $27 \%$ for occupational performance and $40 \%$ for satisfaction with performance.

Conclusion: The findings raise questions regarding what the team might learn from different ways of scrutinizing results; the relevant level of MID in this program; and the overall objective in terms of the proportion of clients who reported a 'successful' outcome in occupational performance and satisfaction with performance, based on the identified MID. These questions need to be further analysed and discussed within the professional team.
\end{abstract}

Keywords: Activity, client-centred, clinical outcome, implementation, statistics.

\section{INTRODUCTION}

One major challenge in research is to implement significant findings into clinical practice. All employees are not accustomed to read and interpret statistical analysis or different outcome variables, which may create uncertainty and a sense of inadequacy. One way to facilitate that process might be to discuss statistical findings based on what can be considered the Minimal Important Difference (MID) from a clinical perspective. Thus, outcome from this presented clinical study are described and discussed from different perspectives.

Individuals who avoid activity due to persistent pain experience a higher level of physical disability and distress compared to those who are more active [1]. A previous study [2] described the consequences of long term pain in terms of decreased performance in daily living and a lower activity pattern. However, the consequences are seldom described in relation to the individuals' needs, wishes and demands.

*Address correspondence to this author at the Clinical Department of Rehabilitation Medicine, University Hospital, SE-581 85 Linköping, Sweden; Tel: +46 01010315 60; Fax: +46 01010315 64;

E-mail: Kersti.Samuelsson@lio.se
Accepting the persistent pain, making rules for living with the pain and developing coping strategies contribute to the individuals' ability to move forward and set goals [3, 4].

Rehabilitation for individuals of working age who experience complex pain over a long period has been found to be most effective when behavioural medicine forms the basis for interventions provided by a multidisciplinary rehabilitation team [5]. For example, cognitive behavioural therapy (CBT) has been found to be more effective than traditional pain management treatment $[6,7]$. The positive effects presented in these studies were expressed as reduced absenteeism and fewer health care visits. Multidisciplinary rehabilitation programs have also been shown to be more cost effective compared to a program based on orthopaedic manual therapy and exercise [8]. Furthermore, many health care organisations highlight the importance of making the patient an active member of the rehabilitation team in terms of guiding the intervention process, taking more responsibility and playing a vital role in its evaluation. With this kind of approach, health care professionals become more like coaches and facilitators, using their professional competence to support the requirements, will and needs of each patient. Due to the heterogeneity of patient 
characteristics and individualized requirements, outcomemeasurement tools must be sufficiently flexible and sensitive for evaluation of a rehabilitation program. Client-Centred Practice (CCP), as described by Sumsion [9], focuses on the client, aims at enabling activity and should be consistent with the challenge of planning and evaluating pain rehabilitation program. The most important characteristic of the CCP process is that the client identifies performance problems and sets the intervention goals. The partnership between the therapist and the client is essential for attaining the agreed goals. Basic assumptions are that clients are experts on their own occupational function and have the right to receive information that supports them in decisionmaking [10]. Clients and therapists work together to define the occupational performance problems, the focus and goal of the intervention as well as the desired outcomes [11]. One of the therapist's roles is to encourage the client to recognise and build on his/her strengths, thus it is important to be aware of the client's insight into his/her condition and previous experiences of the problems as well as to have a mutual understanding of the goals formulated [11].

\section{PAIN MANAGEMENT PROGRAM}

Multidisciplinary pain management programs based on Cognitive Behavioral Therapy (CBT) have been provided for many years to patients with persistent pain at the Pain and Rehabilitation Centre, University Hospital, Linköping, Sweden. A group of eight patients are included in each pain management program. Inclusion criteria are: all relevant medical investigations and examinations must be completed and the patient should have a reasonable opportunity of returning to work or studies. He/she should be strong enough to participate in the program and have a desire and willingness to change his/her current situation. Exclusion criteria are: abuse of alcohol or other drugs, depression or concurrent treatment provided by another caregiver. Adjustments to the program are made on the basis of individual needs and goals. Each subject has his/her own personal physician and a coach (occupational therapist, physiotherapist, psychologist or medical social worker). The program includes group lectures on different themes such as pain management, the importance of physical activity, stress and how to cope with it, sleep, concentration and memory. The lectures are combined with practical individual and group sessions aimed at increasing knowledge and supporting the integration of theory and practice.

The program lasts for 8 weeks and each patient is scheduled for occupational therapy, physiotherapy, group therapy sessions as well as medical information. All members of the multidisciplinary team meet regularly to evaluate the ongoing process and although each profession has its own area of responsibility, they have a common approach, using the CBT as a theoretical base for the intervention. All team members have basic training in CBT, while the occupational therapists are supervised by a CBTtrained psychologist on a regular basis. The occupational therapists have overall responsibility for elucidating questions about occupational performance, including return to work. The occupational therapy intervention offers a choice of three areas: balance in activity patterns; leisure and everyday life; and work/study. The overall goals of occupational therapy are to help individuals live a meaningful life in accordance with their wishes and needs, to balance these against the demands of the environment, to achieve optimal performance in activities that are important to the individuals concerned, to increase patient satisfaction with occupational performance rather than to expect an improvement considering pain, and to make individuals ready for work and/or study. Theory is combined with group discussions shaped by CBT, as described by Linton [12], and the participants are given homework related to occupational performance. The occupational therapists use the Canadian Occupational Performance Measure (COPM) to identify occupational performance problems before interventions and to evaluate the effects of interventions on occupational performance and satisfaction with performance [10]. The weekly group sessions, led by a medical social worker (with CBT education), are intended to influence dysfunctional emotions, behaviours and cognitions by means of a goaloriented, systematic procedure based on cognitive behaviour therapy. Physiotherapy includes basic body knowledge, keep-fit exercises and practical relaxation exercises. Thus, the entire program is based on CBT theories, and interventions are inspired by, among others, the work of Steven Linton [6-7, 12]. At the end of the pain management program, COPM is used as one of the outcome measures [10]. A follow-up is performed 6 months after the end of the program.

\section{THE CANADIAN OCCUPATIONAL PERFORMANCE MEASURE - COPM}

The COPM is a client-centred outcome measure based on the Canadian Model of Occupational Performance, CMOP. The significant characteristic of the CMOP is the interdependence between person, environment and occupation. The environment refers to the physical, social, cultural and institutional environment, all of which are considered to have great influence on occupational performance [13]. Activities take place in the interaction between the person and his/her environment. Spirituality is the central core of the CMOP, embedded in all interactions between person, environment and occupation, as it resides in the person, is shaped by the environment and, above all, gives meaning to activities. Meaning is considered a very important factor for understanding the relationship between occupational engagement, health and well-being. Some occupational scientists position occupational meaningfulness within the framework of spirituality [14]. Meaning is considered a driving force behind as well as an outcome of occupational engagement and accordingly of central importance in all rehabilitation [15].

When using the COPM as an outcome measure, patients evaluate their occupational performance and satisfaction with performance on the basis of their defined problems in the areas of self-care, productivity and leisure [10]. A semistructured interview is conducted to encourage them to identify and discuss specific activities that are difficult to perform. Clients are always prompted to identify activities that they want, need or are expected to do. Once the specific problem areas have been identified, the patient is asked to rate the importance to him/her of each of these activities on a cue card with scores ranging from 1 to $10(1=$ not important at all and $10=$ extremely important). The importance rating is used to prioritise the five most serious problems. The 
patient is then asked to rate his/her ability to perform these specified activities as well as his/her satisfaction with performance using two other cue cards scored from 1 to 10 ( $1=$ not able to do it/not at all satisfied and $10=$ able to do it extremely well/extremely satisfied). The performance and satisfaction scores are then added up separately and divided by the number of problem areas. This calculation provides mean performance and satisfaction scores. Treatment planning is based on the identified problems, with the goal of improving performance in the areas most meaningful for the client. Following an agreed period of intervention, the performance and satisfaction scores are reassessed. The difference between the initial and subsequent scores is the outcome [10].

Several studies in different settings indicate that the COPM is a reliable [16-18], valid [19-21], clinically useful and responsive outcome measure for evaluation of interventions [22-27]. The COPM is considered to have satisfactory external validity and responsiveness to change in patients with chronic low-back pain [28]. Findings from that study showed that approximately one third of the patients reported improvements of two or more points as measured by the COPM [28]. Carpenter et al. [21] tested the COPM as an outcome measure for a pain management program and demonstrated convincing evidence of concurrent criterion validity as well as sensitivity to change. Persson et al. [29] found that changes in occupational performance, psychological well-being and psychosocial functioning are relevant aspects that require assessment and that the COPM is suitable for evaluation of a pain management program. A pilot study of a pain coping strategy program compared chronic pain patients' mood, functional status and physical ability pre and post participation and found a statistically significant improvement on the COPM for both perceived performance and perceived satisfaction, signifying an improvement in the patients' perception of activities of daily living [30]. Irrespective of medical interventions, the early intervention program seemed to be effective in promoting self-management and positive coping strategies.

To our knowledge, there are a few studies (mentioned above) that used the COPM as outcome measure for evaluation of pain management programs. Only one of them include a follow up over time after completion of the program [28]. Thus, there is a need of more knowledge regarding long term effectiveness of pain management programs from a patient perspective. The aim of this study was to describe the effect of a multidisciplinary pain management program in terms of patients' reported occupational performance and satisfaction with performance.

\section{METHODS}

\section{Design}

The design is a retrospective case series study with repeated measurements based on data collected from already existing information in medical records 1) at introduction of the pain management program, 2) at the end of the program, and 3) six months after completion of the program.

\section{Material}

Included were all subjects who participated in a 2-month multidisciplinary pain management program during a one- year period $(n=85)$. Two patients did not attend the second interview, while a further six did not appear for the third interview. Therefore, the number of drop-outs was eight. The final intervention group comprised 77 individuals, 15 men $(18 \%)$ and 62 women. The mean age was 37 years, with a range of 21-56. The pain duration had a median value of 47 months (range 2-325 months). A majority of the patients included in the program had pain related to musculoskeletal problems as defined by the ICD-10 classification [31]. The following ICD-10 diagnoses were identified; M79.0-2 'Rheumatism, unspecified', 'Myalgia' or 'Neuralgia and neuritis, unspecified' $(\mathrm{n}=31)$; M50-54 'Cervical disc disorders', 'Other intervertebral disc disorders', 'Other dorsopathies, not elsewhere classified' or 'Dorsalgia' $(n=12)$; S13.4 'Sprain and strain of cervical spine' $(n=10)$; T91.8 'Sequelae of other specified injuries of neck and trunk' (n=8); R52.9 'Pain, unspecified' (n=7); G44.2 'Tension-type headache' $(n=3)$; mixed diagnoses $(n=14)$. Three clients had no diagnosis.

\section{Data Collection}

Data from the COPM interviews were used as outcome in this study. The COPM [10] is routinely employed both for intervention planning and as an outcome measure for the whole multidisciplinary pain management program. Each patient was interviewed by an occupational therapist prior to the start of the pain management program, in order to identify significant occupational performance problems related to the pain. The most important were defined as goals for the subsequent program. Having defined his/her occupational performance goals, the patient prioritised the list of activities by estimating the importance of each of them. After discussing the prioritization and reaching consensus with the interviewer, each patient rated his/her performance of the most important activities on the two 1-10 point scores for performance and satisfaction with performance [10]. Mean values for these scores were calculated. At the end of the multidisciplinary pain management program and six months later, the patients were again asked to estimate their performance and satisfaction with performance. The patients were not allowed to look at the former estimations on these occasions. Information from the patients' medical records regarding sick leave before the program and 6 months after was collected.

\section{Ethical Considerations}

Since this is a clinical study based on data from existing documentation and medical records, and no treatment or data were added to the standard program, the study did not require approval from an ethics committee in accordance with Swedish law [32]. All data from the medical records including the COPM-data were collected by a research nurse, registered in an Excel file and delivered to the authors without identifiable information. It is thus not possible to identify individual results.

\section{Statistics}

Statistica 7.0 was used for all statistical analyses including; the Wilcoxon matched pair test, Friedman's test, Kendall's Coefficient of Concordance and Spearman's rank correlation coefficient. Mean values were calculated based on each individual's occupational performance and 
satisfaction with performance scores. The differences in mean values from the three data collection occasions were analysed both from a statistical and from a clinical perspective. A $p$-value of $<0.01$ was set as an acceptable level of significance in the statistical analysis, and a difference of at least 2 points in a patient's mean score was considered clinically relevant and important [10].

\section{RESULTS}

The initial COPM interviews, which were conducted by one occupational therapist working at the department, prior to the start of the program, resulted in the identification of 652 highly prioritized occupational performance problems, which consequently constituted the basis for rehabilitation interventions and evaluation of the program. At the 6-month follow-up, 555 of those problems were still present as reported by the 77 patients who attended on all three data collection occasions. In order to determine the most frequently identified activities, all activities were placed into categories and subcategories in accordance with the instructions and structure of the COPM manual [10]. The categorization of activities was performed by one person not involved in the pain management program or in data analysis. The most frequently mentioned occupational problems from the first interview were found in the categories of productivity and leisure. At the top of the list was household management (31\%), followed by active recreation (15\%) and functional mobility (12\%) (Fig. 1). Household management included activities such as cleaning, cooking, shopping and taking care of the family. Active recreation involved activities such as exercising, walking and gardening. Functional mobility comprised activities such as transportation and driving. The most frequently prioritised individual occupational performance problems were related to the ability to work (mentioned by $69 \%$ of patients) followed by exercising.

The overall mean value for performance on the three data collection occasions was; before the program $\mathrm{m}=4.7$, after the program $m=5.2$ and at the 6 -month follow-up $m=5.6$. The analysis of variance showed a difference between measures, $\mathrm{p}<0.001$ (Table 1). For satisfaction with performance, the overall mean value was; before the program $\mathrm{m}=3.7$, after the program $\mathrm{m}=4.8$ and at the 6-month follow-up $\mathrm{m}=5.3$. The analysis of variance also revealed a difference between measures, $\mathrm{p}<0.001$ (Table 2). There was an improvement over time in occupational performance as well as satisfaction with performance, as measured by the Wilcoxon matched pair test, when the overall mean values for each subject prior to the start of the multidisciplinary pain management program were compared to those at the first and 6-month follow-up (Table 3). The difference in scores after the program versus the 6-month follow-up was only significant for occupational performance $(\mathrm{p}<0.01)$.

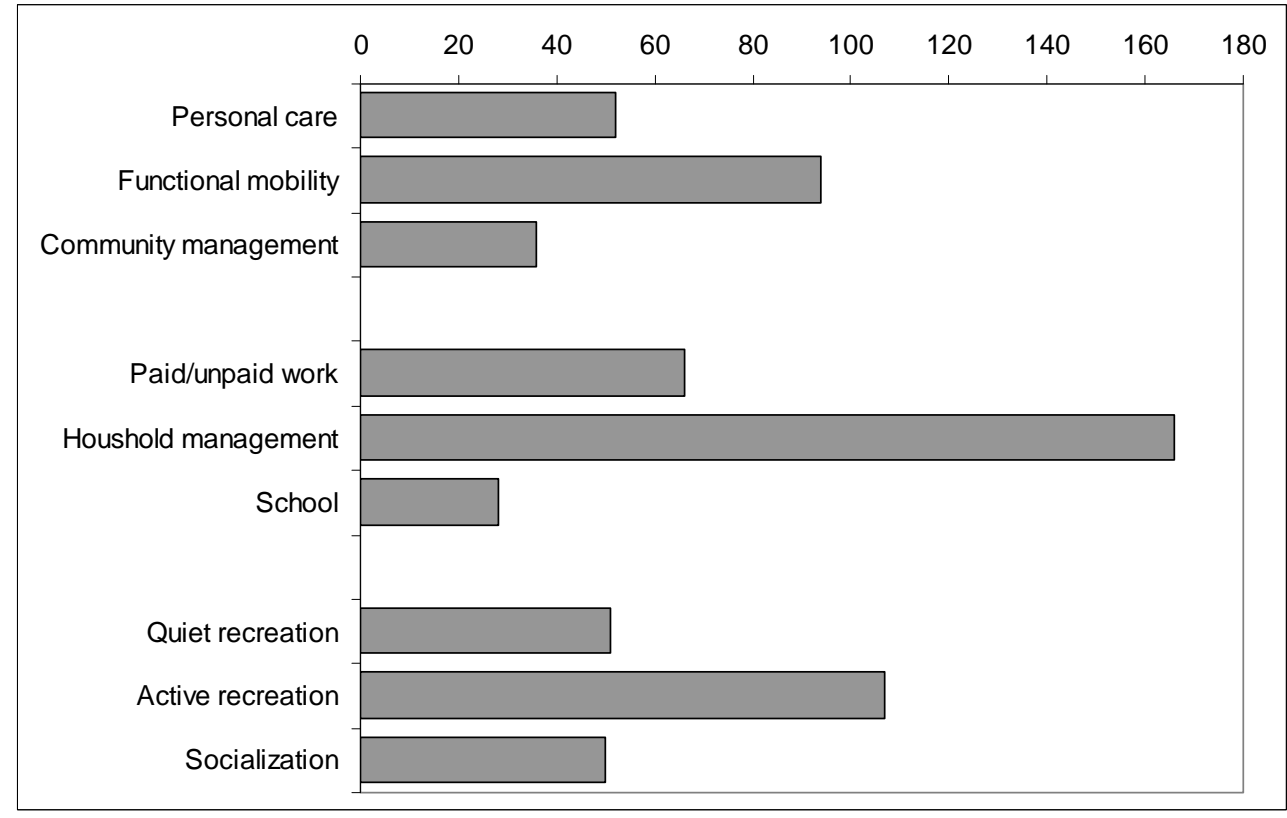

Fig. (1).. Number of prioritized problems in each sub-category as identified at the beginning of the program (total number of identified occupational problems $=652$ ). The most common problems could be categorised as household management followed by active recreation and functional mobility.

Table 1. Comparison Between Mean Estimated Occupational Performance from the Three Different Data Collection Occasions.

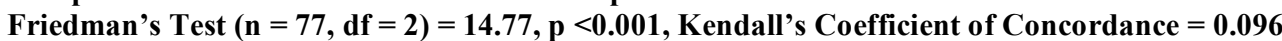

\begin{tabular}{|c|c|c|c|}
\hline Variable & Average Rank & Sum of Ranks & Mean \\
\hline \hline Occupational performance before & 1.73 & 133.000 & 1.69 \\
\hline Occupational performance after & 1.94 & 1.18 & 5.15 \\
\hline Occupational performance at 6-month follow-up & 2.33 & 179.500 & 5.63 \\
\hline
\end{tabular}


Table 2. Comparison between Estimated Satisfaction with Occupational Performance from the Three Different Data Collection Occasions. Friedman's Test $(\mathrm{n}=77, \mathrm{df}=2)=28.51 \mathrm{p}<0.001$, Kendall's Coefficient of Concordance $=0.185$.

\begin{tabular}{|c|c|c|c|c|}
\hline Variable & Average Rank & Sum of Ranks & Mean & SD \\
\hline Satisfaction with occupational performance before & 1.54 & 118.500 & 3.68 & 1.41 \\
\hline Satisfaction with occupational performance after & 2.08 & 160.000 & 4.75 & 1.81 \\
\hline Satisfaction with occupational performance at 6-month follow-up & 2.38 & 183.500 & 5.33 & 2.18 \\
\hline
\end{tabular}

Table 3. The p-Value Represents the Overall Significant Level of Difference (Wilcoxon Matched Pairs Test). The Percentage of Patients $(n=77)$ having a Mean Difference in Estimated Occupational Performance Versus Satisfaction with Performance, between the Three Data Collection Occasions

\begin{tabular}{|c|c|c|c|c|c|}
\hline Occupational Performance & p-Value & $\begin{array}{c}\text { Mean Difference Score } \\
\leq 0\end{array}$ & $\begin{array}{c}\text { Mean Difference Score } \\
>0-<1\end{array}$ & $\begin{array}{c}\text { Mean Difference Score } \\
\geq 1 \leq 2\end{array}$ & $\begin{array}{l}\text { Mean Difference } \\
\text { Score }>2\end{array}$ \\
\hline Before $v s$ after the program & 0.008 & $40 \%$ & $25 \%$ & $25 \%$ & $10 \%$ \\
\hline After $v s$ at 6-month follow-up & 0.006 & $36 \%$ & $21 \%$ & $30 \%$ & $13 \%$ \\
\hline Before $v s$ at 6-month follow-up & $<0.001$ & $31 \%$ & $18 \%$ & $23 \%$ & $27 \%$ \\
\hline \multicolumn{6}{|c|}{ Satisfaction with Occupational Performance } \\
\hline Before $v s$ after the program & $<0.001$ & $30 \%$ & $19 \%$ & $19 \%$ & $31 \%$ \\
\hline After $v s$ at 6-month follow-up & $0.016(\mathrm{~ns})$ & $40 \%$ & $19 \%$ & $18 \%$ & $22 \%$ \\
\hline Before $v s$ at 6-month follow-up & $<0.001$ & $23 \%$ & $16 \%$ & $21 \%$ & $40 \%$ \\
\hline
\end{tabular}

From a clinical perspective, a Minimal Important Difference (MID) of at least 2 points on the COPM is considered clinically relevant [10]. Our results showed that $10 \%$ of clients $(n=8)$ had a mean difference of at least 2 points in occupational performance before compared to immediately after the program and that the corresponding figure for occupational performance before the program versus the 6-month follow-up was $27 \%(\mathrm{n}=21)$. Regarding satisfaction with performance, a difference of at least 2 points was found in 24 clients (31\%) when comparing before versus the end of the program, and in 31 clients $(40 \%)$ when comparing the mean scores before the program versus the 6month follow-up (Table 1). An analysis of the pair-wise differences between each individual's scores on performance versus satisfaction with performance at the three different measurements revealed a difference at baseline $(p<0.001)$ as well as after the conclusion of the program $(p<0.001)$ but not at the final follow-up $(\mathrm{p}=0.02)$. Thus, satisfaction with performance was rated lower than performance before and directly after the program.

\section{Correlation Between Measurements}

The correlation between scores for performance and satisfaction with performance increased over time. Before the pain management program the correlation coefficient was $\mathrm{r}_{\mathrm{s}}=0.59$. At the end of the program, the correlation coefficient between performance and satisfaction was $r_{s}=$ 0.78 and, at the 6-month follow-up, $r_{s}=0.89$ (Fig. 2A-C).

\section{Sick-Leave}

Before the pain management program started, $46 \%$ of the patients were on $100 \%$ sick-leave and $18 \%$ in full-time work or studies (Table 4). At follow-up 6 months after the program, $24 \%$ were on $100 \%$ sick-leave and $39 \%$ in fulltime work or studies, a significant difference $(\mathrm{p}<0.001)$.

Table 4. Percentage of Patients on Sick-Leave, before and at the Follow-Up 6 Months after the Pain Management Program (n=85). Chi ${ }^{2}$ Test. $p<0.001$

\begin{tabular}{|c|c|c|}
\hline Sick-Leave, $\%:$ & Before $\%$ & Follow Up \% \\
\hline \hline $0 \%$ & 18 & 39 \\
$25 \%$ & 10 & 1 \\
$50 \%$ & 22 & 27 \\
$75 \%$ & 4 & 9 \\
$100 \%$ & 46 & 24 \\
\hline
\end{tabular}

\section{DISCUSSION}

This study aims to describe the outcome of a pain management program by focusing on patient reported occupational performance and satisfaction with performance. The main and overall findings reveal that performance as well as satisfaction with performance improved for this group of patients after having participated in the program. However, from a clinical perspective, the difference only pertained to $27 \%$ of the patients (at least 2 points difference over time), when comparing data from before the program versus the 6 month follow-up. The findings on satisfaction with performance were similar, although the results were slightly better and $40 \%$ of the patients had a difference of at least 2 points. Our results confirm the findings of Walsh et al. [28]. 

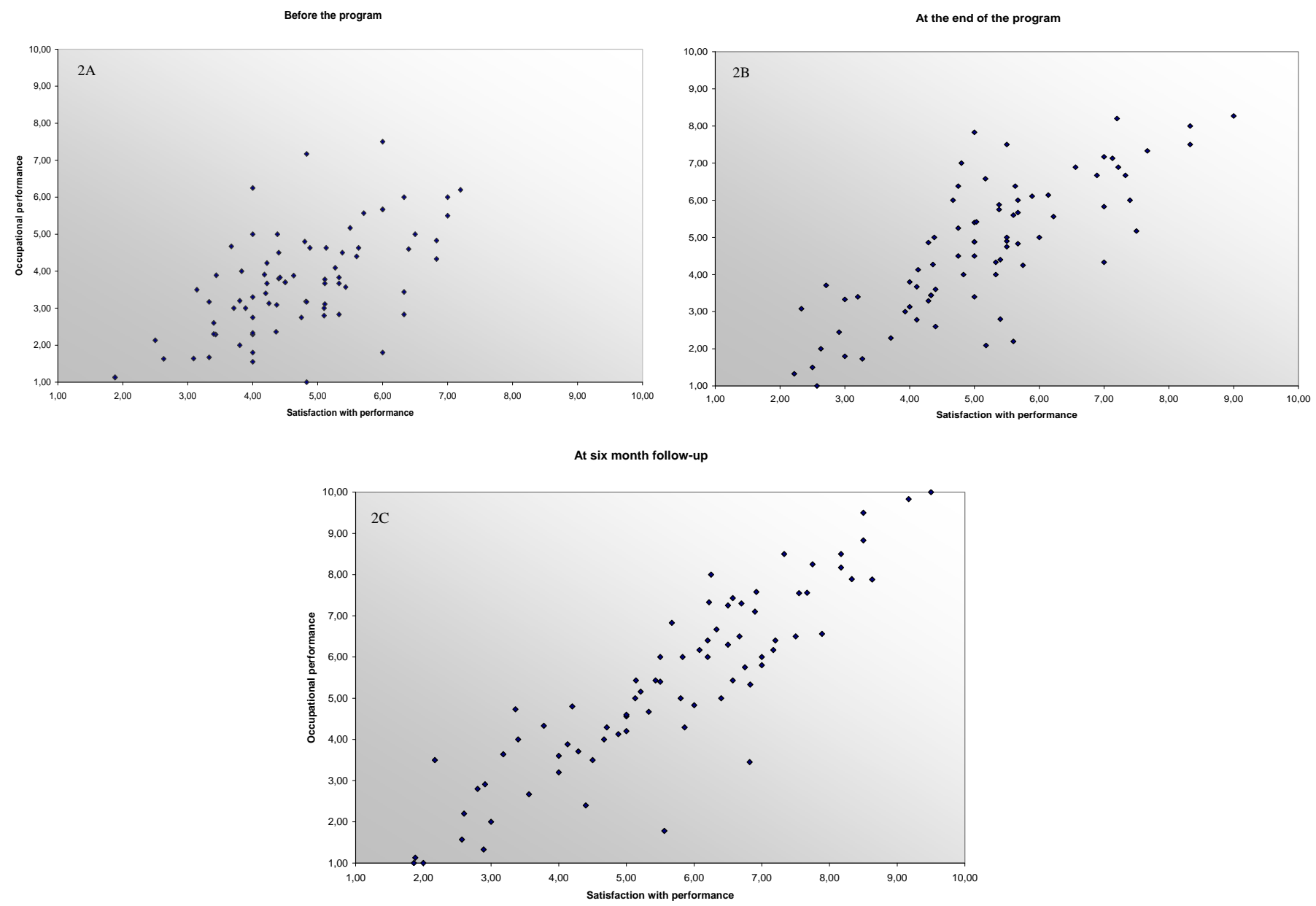

Fig. (2). (A-C) Scatter plots for occupational performance and satisfaction with performance scores; before and after the pain management program and at the 6-month follow-up. Before the intervention, the correlation coefficient was $r_{s}=0.59$. After the intervention, $r_{s}=0.78$ and at follow-up 6 months after the program $\mathrm{r}_{\mathrm{s}}=0.89$.

Before the intervention, the estimated overall mean score for satisfaction with performance was significantly lower than that for performance, which could be expected. Even at the end of the program, satisfaction was still rated lower than performance, although the difference was less. Above all, satisfaction with performance improved over time, and at the six-month follow-up, there was no longer a significant difference between satisfaction with performance and performance scores. This is an interesting and positive result, as the overall goal of the multidisciplinary pain management program is to help patients to adjust to a life with undesirable restrictions by increasing their physical capacity, optimising their activity level and, above all, improving their satisfaction with life. The results were mainly influenced by the fact that the patients had learned individual pain management strategies that were effective over time, which requires further investigation. According to the therapists working within the department, patients taking part in the program gain the ability to verbally express the changes in their life situation and, when they accept their situation, will be able to move on with their lives and cope with the pain and associated restrictions. Coping strategies encompass analysing and adapting to the situation as well as learning to live with pain. The overall results from this study also confirm some of the findings from other studies [29-30, 33, 34].
Three main questions could be raised from these results; what can the clinical department learn from the different ways of analysing and scrutinizing results? What would be a relevant level of Minimal Important Difference (MID) in this particular program? What is a relevant overall objective for this program, in terms of the proportion of clients who reported a 'successful' outcome in occupational performance and satisfaction with performance, based on the identified MID? Results indicated that the program was significantly effective, both for occupational performance and for satisfaction with performance. However, if the minimal level of improvement is set at $\geq 2.0$ points on the 10 -point scale, 'only' $40 \%$ of the patients are more satisfied with performance after 6 months compared to before the program, and less than $30 \%$ consider that they can perform the individually prioritized activities better 6 months after, compared to before the program. Is this result good enough? If not, what result is to be expected/desirable? This question still remains to be discussed within the professional team together with the question about the Minimal Important Difference in COPM outcome scores. According to the manual [10] a difference of two points should be considered as clinical important, this cut off is normally used in published papers [28]. Future clinical practice could benefit from using COPM as an outcome measure in combination 
with other kinds of outcome data in order to facilitate a clinical discussion of this matter.

In clinical practice, the COPM has been found to help patients identify and frame their problems and evaluate their own performance $[3,19]$, thus engaging them in rehabilitation planning $[21,35,36]$. The instrument facilitates clientdefined goals $[25,37,38]$. Patients are helped to focus on themselves [39] and develop competence [35]. It also provides more nuanced information about patient preferences than other standardized outcome measures [23, 39]. Thus, interventions are based on problems identified by the patient, and outcome measures are indicated by the COPM scores, which are provided by the patient him/herself.

Household activities were often identified as problematic within the patient group. A majority of included patients were women $(82 \%)$ and this picture might look different with more men in the group. However, no separation of men and women regarding what kind of activities that are perceived to be most problematic has been made in this study, since this was no aim in the study.

Using mean value to obtain a summarised value for each individual's estimated performance and satisfaction could be questioned. Although the activities are individually identified and therefore vary between clients, the mean value should be considered as the estimated overall level of occupational performance and satisfaction with performance. Additionally, according to the COPM manual, the mean value is recommended in the data analysis [10]. We used non-parametric statistics for the analyses, as this method was deemed suitable for our data. Based on results from our study, we suggest that the COPM could be a relevant and useful evaluation tool in clinical practice, not only as a basis for an overall discussion about the goal of the program but also at individual level when planning for and evaluating results from different interventions.

All interventions as well as the patients' own learning processes certainly contributed to the overall results. Ergonomic interventions and adaptations of the environment, in addition to body knowledge and the importance of being physically active, are examples of the content of the pain management program. It was not possible to identify one single factor as responsible for the increased performance and satisfaction with performance. The use of cognitive behaviour therapy as a cornerstone and core concept of the multidisciplinary pain management program is likely to have permeated the whole intervention with a key contribution to the positive results.

Existing research has already shown beneficial effects of multidisciplinary approaches (e.g. biopsychosocial approaches) compared to traditional orthopaedic and pain management rehabilitation in terms of coping and depression [5]. Linton and Ryberg [6] randomly assigned 253 participants with severe back pain during the previous year to either a CBT group intervention or usual treatment. At a one-year follow up, the CBT group had better results on 26 out of 33 variables compared to the control group. The CBT group also had a threefold-reduced risk of long-term sick leave. Similar results were presented by Linton et al. [7]. In a recent study, Jensen et al. [8] illustrated the high cost effectiveness of a multidisciplinary program compared to a more traditional program with manual therapy and physical exercise. However, few results have been presented in terms of improvement in patients' own identified activity and participation problems or in the light of Minimal Important Difference. In a review by Turk et al. [40], the authors concluded that there is a lack of patient reported outcome measures for clinical pain trials. The authors highlighted the importance of relevant outcome variables for defining what should be considered successful results and how best to measure them.

Accepting pain as a part of life and developing active coping strategies lead to reduced psychological distress [1, 3] and high levels of daily functioning [4]. The program increased the participants' occupational performance and satisfaction with their performance, which may have enhanced their psychological well being. Results from this and other studies suggest that aspects of psychological wellbeing and psychosocial functioning must be addressed together with occupational performance [29] and that improvements in performance and satisfaction with performance are associated with increased self-efficacy and improved observed performance [28]. Accordingly, promoting self-management and teaching positive strategies seem to be effective for long-term pain patients, which is supported by our study as well as other studies [30, 41]. A multidisciplinary pain management program should address the multiple factors that influence pain management and coping skills [42], where the key to success is considered to be based on ongoing communication between team members and the patient [43].

\section{Study Limitations}

It would have been preferable to include a control group but this was not possible since it is a retrospective clinical study and the purpose was to explore current clinical practice. Thus, we cannot conclude that the results are solely due to the multidisciplinary pain management program, as other factors may have contributed to the reported outcome. The fact that the COPM interviews were performed by one of the occupational therapists in the rehabilitation team could be considered a limitation, and it would have been preferable to have an independent interviewer in order to ensure reliability. However, COPM is based on a client-centred approach in which the client is responsible for defining individually prioritised problems and goals as well as the occupational performance and satisfaction scores. The therapist's role is solely to facilitate the process for the individual.

Performing outcome studies based on clinical data from medical records is a challenge. Data are often missing and/or collected without sufficient structure and reliable methods. Clinical circumstances sometimes make it impossible to select the 'right' patients for a special study purpose, and outcome data frequently have to be collected by persons who are engaged in the patients' treatment. At the same time, health professionals are required to provide the most efficient medical care. Since there is a general lack of clinical evidence in many medical specialities and especially in the area of rehabilitation medicine, this is a dilemma. Rehabilitation medicine often includes an unspecified mixture of individually planned and evaluated interventions. 
However, in striving to use the best possible evidence and develop clinical guidelines, the Pain and Rehabilitation Centre at Linköping University Hospital has established standard routines and registers to ensure the best possible data for outcome evaluation, thus making this retrospective study possible.

\section{CONCLUSION}

The Canadian Occupational Measure (COPM) has been shown to be a useful tool in the planning and evaluation of a multidisciplinary pain management program, taking patients' individual wishes and needs into account. A comparison of the mean values for all subjects at the start of the program with mean values at the first and second follow-ups revealed an improvement over time. Above all, the results demonstrate that satisfaction with performance increases over time, which was an overall aim of the program. Future studies including a control group are necessary to verify the present results. The findings raise questions regarding what the team might learn from different ways of scrutinizing results; the relevant level of MID in this program; and the overall objective in terms of the proportion of clients who reported a 'successful' outcome in occupational performance and satisfaction with performance, based on the identified MID. These questions need to be discussed within the professional team together with the question about the Minimal Important Difference in COPM outcome scores.

\section{DECLARATION OF INTEREST}

The authors report no conflict of interest. The authors alone are responsible for the content and writing of the paper.

\section{REFERENCES}

[1] McCracken L, Samuel V. The role of avoidance, pacing, and other activity patterns in chronic pain. Pain 2007; 130(1): 119-25.

[2] Spenkelink CD, Hutten MM, Hermens HJ, Greitemann BO. Assessment of activities of daily living with an ambulatory monitoring system: a comparative study in patients with chronic low back pain and nonsymptomatic controls. Clin Rehabil 2002;16(1): 16-26.

[3] Clarke KA, Iphofen R. Accepting pain management or seeking pain cure: an exploration of patients' attitudes to chronic pain. Pain Manag Nurs 2007; 8(2): 102-10.

[4] Ramírez-Maestre C, Esteve R, López A. Cognitive appraisal and coping in chronic pain patients. Eur J Pain 2008; 12(6): 749-56.

[5] Mangels M, Schwartz S, Worringen U, Holem M, Rief W. Evaluation of a behavioral-medical inpatient rehabilitation treatment including booster sessions: a randomized controlled study. Clin J Pain 2009; 25(5): 356-64.

[6] Linton SJ, Ryberg M. A cognitive-behavioral group intervention as prevention for persistent neck and back pain in a non-patient population: a randomized controlled trial. Pain 2001; 90(1-2): 8390.

[7] Linton SJ, Boersma K, Jansson M, Swärd L, Botvalde M. The effects of cognitive-behavioral and physical therapy preventive interventions on pain-related sick leave. Clin J Pain 2005; 21(2): 109-19.

[8] Jensen IB, Busch H, Bodin L, Hagberg J, Nygren A, Bergström G. Cost effectiveness of two rehabilitation programmes for neck and back pain patients: a seven year follow-up. Pain 2009; 142(3): 2028.

[9] Sumsion T. Client-centred practice in occupational therapy: a guide to implementation. Philadelphia PA: Churchill Livingstone 2000.

[10] Law M, Baptiste S, Carswell A, McColl MA, Polatajko H, Pollock N. Canadian occupational performance measure. $4^{\text {th }}$ ed. Toronto ON: CAOT Publications ACE 2005.

[11] Baum CM, Law M. Occupational therapy practice: Focusing on occupational performance. Am J Occup Ther 1997; 51(4): 277-88.
[12] Linton S. Cognitive-Behavioral Therapy in the early treatment and prevention of chronic pain. Hallsberg, Sweden: NärkeTryck AB 2000.

[13] Polatajko H, Backman C, Baptiste S et al. Human occupation in context. In Townsend E, Polatajko H, Eds. Enabling Occupation II. Advancing an occupational therapy vision for health, well-being \& justice through occupation. Toronto ON: CAOT publications ACE 2007.

[14] Townsend E, Ed. Enabling occupation: An occupational therapy perspective. 2nd ed. Ottowa: CAOT Publications ACE 2002.

[15] Persson D, Erlandsson L, Eklund M, Iwarsson S, Value dimensions, meaning, and complexity in human occupation - a tentative structure for analysis. Scand J Occup Ther 2001; 8(1): 7-18.

[16] Sewell L, Singh SJ. The Canadian Occupational Performance Measure: Is it a reliable measure in clients with chronic obstructive pulmonary disease? Br J Occup Ther 2001; 64(6): 305-10.

[17] Cup EHC, Reimer WJM, Thijssen MCE, Van Kuyk-Minis MAH. Reliability and validity of the Canadian Occupational Performance Measure in stroke patients. Clin Rehabil 2003; 17(4): 402-9.

[18] Pan A-W, Chung L, Hsin-Hwei G. Reliability and validity of the Canadian Occupational Performance Measure for clients with psychiatric disorders in Taiwan. Occup Ther Int 2003; 10(4): 26977.

[19] McColl MA, Paterson M, Davies D, Doubt L, Law M. Validity and community utility of the Canadian Occupational Performance Measure. Can J Occup Ther 2000; 67(1): 22-30.

[20] Chan CCH, Lee TMC. Validity of the Canadian Occupational Performance Measure. Occup Ther Int 1997; 4(3): 229-47.

[21] Carpenter L, Baker GA, Tyldesley B. The use of the Canadian Occupational Performance Measure as an outcome of a pain management program. Can J Occup Ther 2001; 68: 16-22.

[22] Wressle E, Marcusson J, Henriksson C. Clinical utility of the Canadian Occupational Performance Measure - Swedish version. Can J Occup Ther 2002; 69: 40-8.

[23] Wressle E, Samuelsson K, Henriksson C. Responsiveness of the Swedish version of the Canadian Occupational Performance Measure. Scand J Occup Ther 1999; 6: 84-9.

[24] Richardson JC, Ong B, Sim J. Experiencing and controlling time in everyday life with chronic widespread pain: a qualitative study. BMC Musculoskeletal Disord 2008, 9: 3.

[25] Chesworth C, Duffy R, Hodnett J, Knight A. Measuring clinical effectiveness in mental health: Is the Canadian Occupational Performance Measure an appropriate measure? Br J Occup Ther 2002; 65(1): 30-34.

[26] Corr S, Wilmer S. Returning to work after a stroke: an important but neglected area. Br J Occup Ther 2003; 66(5): 186-92.

[27] van Leit B, Crow T. Outcomes of an occupational therapy program for mothers of children with disabilities: Impact on satisfaction with time use and occupational performance. Am J Occup Ther 2003; 56: 402-10.

[28] Walsh DA, Kelly SJ, Johnson PS, Rajkumar S, Bennetts K. Performance problems of patients with chronic low-back pain and the measurement of patient-centered outcome. Spine 2004; 29(1): 87-93.

[29] Persson ER, Rivano-Fischer M, Eklund M. Evaluation of changes in occupational performance among patients in a pain management programme. J Rehabil Med 2004; 36: 85-91.

[30] Mead K, Theadom A, Byron K, Dupont S. Pilot study of a 4-week Pain Coping Strategies (PCS) programme for the chronic pain patient. Disabil Rehabil 2007; 29(3): 199-203.

[31] WHO. International Classification of Diseases ICD-10 Online. Available from: http://apps.who.int/classifications/apps/icd/icd10on line/

[32] Svensk Författningssamling. Lagen om etikprövning av forskning som avser människor. SFS 2003: 60. Available from: http://riksdagen.se/webbnav/index.aspx?nid=3911\&bet=2003:460

[33] Man AC, Chu MC, Chen PP, Ma M, Gin T. Clinical experience with a chronic pain management programme in Hong Kong Chinese patients. Hong Kong Med J 2007; 13(5): 372-8.

[34] Dysvik E, Nativik GK, Eikeland OJ, Brattberg G. Results of a multidisciplinary pain management program: a 6- and 12-month follow-up study. Rehabil Nurs 2005; 30(5): 198-206.

[35] Healy H, Rigby P. Promoting independence for teens and young adults with physical disabilities. Can J Occup Ther 1999; 66(5): 240-9. 
[36] Ripat J, Etcheverry E, Cooper J, Tate RB. Comparison of the Canadian Occupational Performance Measure and the Health Assessment Questionnaire. Can J Occup Ther 2001; 68(4): 248-53.

[37] Kjeken I, Slatkowsky-Christensen B, Kvien TK, Uhlig T. The Norwegian version of the Canadian Occupational Performance Measure in patients with hand osteoarthritis: validity, responsiveness and feasibility. Arthritis Rheum 2004; 51(5): 70915.

[38] Samuelsson K, Tropp H, Gerdle B. Shoulder pain and its consequences in paraplegic spinal cord-injured wheelchair users. Spinal Cord 2004; 42(1): 41-6.

[39] Dedding C, Cardol M, Eyssen IC, Dekker J, Beelen A. The validity of the Canadian Occupational Performance Measure: a clientcentred outcome measurement. Clin Rehabil 2004; 18(6): 660-7.
[40] Turk DC, Dworkin RH, Burke LB et al. Developing patientreported outcome measures for pain clinical trials: IMMPACT recommendations. Pain 2006; 125: 208-15.

[41] Müllersdorf M. Occupational therapists' assessments of adults with long-term pain: The Swedish experience. Occup Ther Int 2002; 9(1): 1-23.

[42] Dysvik E, GuttormsenVinsnes A, Eikeland O-J. The effectiveness of a multidisciplinary pain management programme managing chronic pain. Int J Nurs Pract 2004; 10: 224-34.

[43] Stanos S. Multidisciplinary and interdisciplinary management of chronic pain. Phys Med Rehabil Clin North Am 2006; 17(2): 43550 .

Received: June 29, 2011

Revised: September 12, 2011

Accepted: September 13, 2011

(C) Samuelsson et al.; Licensee Bentham Open.

This is an open access article licensed under the terms of the Creative Commons Attribution Non-Commercial License (http: //creativecommons.org/licenses/by$\mathrm{nc} / 3.0 /$ ) which permits unrestricted, non-commercial use, distribution and reproduction in any medium, provided the work is properly cited. 